Radial and Nonradial Pulsations as Probes of Stellar Physics

ASP Conference Series, Vol. 259, 2002

C. Aerts, T.R. Bedding, \& J. Christensen-Dalsgaard, eds.

\title{
Preliminary Results of a Search for Variable Stars in the Globular Cluster M 13
}

\author{
Grzegorz Kopacki, Zbigniew Kolaczkowski, Andrzej Pigulski \\ Wroctaw University Observatory, Kopernika 11, 51-622 Wrockaw, \\ Poland
}

\begin{abstract}
Results of a search for variable stars in the globular cluster M 13 are presented. Comments on the variability of all observed variable or suspected variable stars are given. Five new RR Lyrae stars and one SX Phoenicis star are identified.
\end{abstract}

\section{Introduction}

We give here the results of a search for variable stars in the central region of the globular cluster M 13. The search was performed with the use of the image subtraction method of Alard \& Lupton (1998).

Our CCD observations were obtained at the Białków station of the Wroclaw University Observatory with a $0.6-\mathrm{m}$ reflecting telescope. A $6 \times 4 \operatorname{arcmin}^{2}$ field of view, covering the core of the cluster, was recorded through $V$ and $I_{\mathrm{C}}$ filters of the Johnson-Kron-Cousins $U B V(R I)_{\mathrm{C}}$ system. The observations were carried out on 22 nights between 2001 February 27 and May 26.

\section{Results}

Prior to this study, 34 variable and suspected variable stars were found in the cluster (Clement, 1997; Osborn, 2000). Of these, nine were not observed by us. Some suspected variables turned out to be in fact constant. We did not detect any light changes in $\mathrm{v} 3, \mathrm{v} 4, \mathrm{v} 12$, and $\mathrm{v} 13$, whereas v10 showed only marginal variability. Only two of the 10 variable-star candidates of Kadla et al. (1980) turned out to be variable. These are v25 and v31, which we classify as RRc variables. Additionally, three new RR Lyrae stars and one SX Phoenicis variable were found. Figure 1 shows the phase diagrams for the two RR Lyrae stars classified by us and the four new variables. The total number of known RR Lyrae stars in M 13 is now nine. Only one of them is an RRab star. M 13 was known to contain three BL Herculis variables. For these stars we also obtained light curves with a good phase coverage.

Almost all bright giants we observed show some degree of variability. In particular, we confirm the variability of four red giants announced to be variable by Osborn (2000) and find five new cases.

The new RRc variable n4 shows clear changes from cycle to cycle (see Fig. 1). Although our observations cover only about 3 months, we were able to derive two closely spaced frequencies for this star. Thus, it appears that $n 4$ 


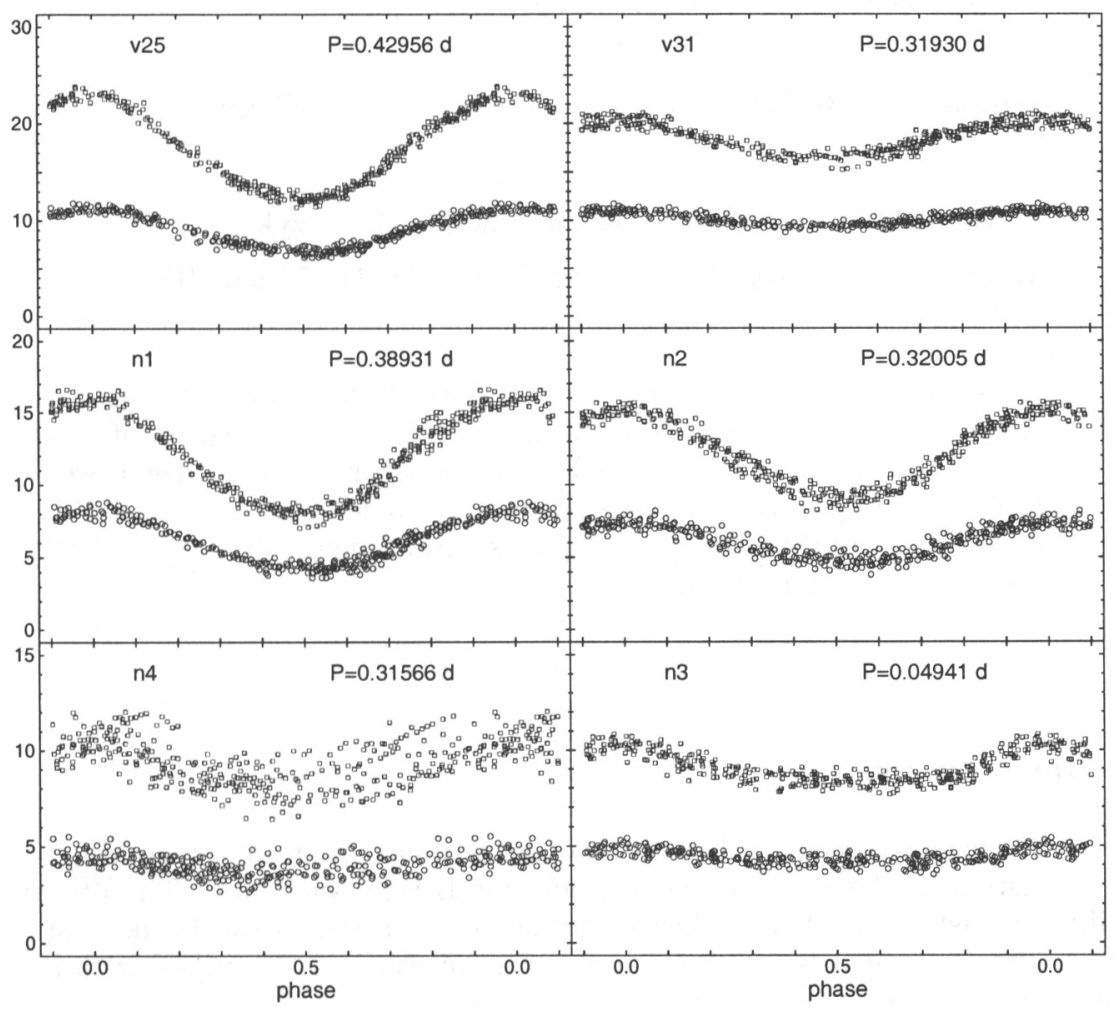

Figure 1. $\quad V$-filter (squares) and $I_{\mathrm{C}}$-filter (circles) light-curves of the new RR Lyrae variables (v25, v31, n1, n2, and n4) and an SX Phoenicis star, n3. The ordinate is expressed in arbitrary flux units. Note different ordinate scales.

belongs to the group of recently discovered double-period RRc variables having a non-radial mode (Olech et al. 1999).

Acknowledgments. This work was supported by KBN grant 2P03D 006 19.

\section{References}

Alard, C. \& Lupton, R.H. 1998, ApJ, 503, 325

Clement, C. 1997, Preliminary Fourth Edition of the Catalogue on Variable Stars in Globular Clusters, electronic version.

Kadla, Z.I., Gerashchenko, A.N., \& Yablokova, N.V. 1980, Astronomicheskii Tsirkular, 1136, 5

Olech, A., Kałużny, J., Thompson, I.B., et al. 1999, AJ, 118, 442

Osborn, W. 2000, AJ, 119, 2902 